Peggy Bosch ${ }^{1,2}$, Sabina Lim ${ }^{3}$, Heike Staudte', Sook-Hyun Lee ${ }^{3}$, Sujung Yeo ${ }^{4}$, Daniela Litscher ${ }^{5}$, Gerhard Litscher ${ }^{5}$, Lu Wang ${ }^{5}$, Maurits van den Noort ${ }^{3,6}$ ${ }^{1}$ Psychiatrische Forschungsgruppe, LVR-Klinik Bedburg-Hau, Bedburg-Hau, Deutschland

${ }^{2}$ Donders Institut für Gehirn, Kognition und Verhalten, Radboud Universität Nimwegen, Nijmegen, Niederlande

${ }^{3}$ Forschungsgruppe für Schmerz und Neurowissenschaften, Kyung Hee Universität, Dongdaemun-gu, Seoul, Südkorea

${ }^{4}$ Abteilung für Orientalische Medizin, Sang Ji Universität, Wonju, Südkorea

${ }^{5}$ Forschungseinheit für biomedizinische Technik in Anästhesie und Intensivmedizin, Forschungseinheit für komplementäre und integrative Lasermedizin und TCM Forschungszentrum Graz, Medizinische Universität Graz, Graz, Österreich

${ }^{6}$ Brüssels Institut für Angewandte Linguistik, Freie Universität Brüssel, Brüssel, Belgien

\title{
Geschlechtsunterschiede bei der Akupunkturbehandlung von Patienten mit Depressionen
}

\begin{abstract}
Hintergrund und Fragestellung
Depression ist eine psychische Störung und zeichnet sich durch eine gedrückte Stimmung, Interessensverlust und wenig Antrieb aus [1]. Die Prävalenz der Krankheit liegt bei Frauen höher als bei Männern [2] und die Symptomatik ist bei Frauen und Männern unterschiedlich ausgeprägt [3]. Als Therapie werden meistens Medikamente [4] und Psychotherapie [5] eingesetzt, aber heutzutage auch immer öfter Akupunktur als Zusatzbehandlung [6]. Das Ziel dieser Studie ist es, zu untersuchen, ob und, wenn Ja, welche Geschlechtsunterschiede es bei der Akupunkturbehandlung von Patienten mit Depressionen gibt. Die primäre Hypothese ist, dass es Geschlechtsunterschiede in den vorhandenen TCM-diagnostischen Mustern und in der Akupunkturbehandlung von weiblichen und männlichen Patienten mit Depressionen gibt.
\end{abstract}

\section{Studiendesign und Untersuchungsmethoden}

\section{Versuchspersonen}

Es wurden 30 Patienten mit Depressionen, 20 Frauen und 10 Männer, über einen Aushang in einer großen deutschen psychiatrischen Klinik angeworben. Alle Patienten standen unter Standardbehandlung (Medikamente, Psychotherapie) in der Klinik, wurden aber nicht stationär in der Klinik behandelt. Die Behandlung war unterschiedlich von Patient zu Patient, was bedeutet, dass nicht jeder Patient die gleiche Medikation bekam. Bei einigen wurden mehr Medikamente verabreicht als bei anderen und nicht alle Patienten waren in psychotherapeutischer Behandlung (da nicht alle dazu bereit waren und auch aus westlicher Sicht unterschiedliche Symptome manchmal zur Gabe von anderen Medikamenten führen). Alle Patienten wurden von ihrem Psychiater nach den ICD-10-Kriterien mit einer rezidivierenden depressiven Störung, gegenwärtig schwere Episode ohne

Deutsche Zeitschrift für Akupunktur 2019 • 62 (3): 160-165 https://doi.org/10.1007/s42212-019-0189-1

Online publiziert: 18. Juli 2019

(c) Der/die Autor(en) 2019 psychotische Symptome, diagnostiziert [1]. Als Ausschlusskriterien galten: Suchterkrankungen, vorherige hirnorganische Erkrankungen (wie Epilepsie oder Parkinson), sonstige psychiatrische Diagnosen (wie Schizophrenie, Persönlichkeits-, oder sonstige mögliche komorbide Störungen) und so weiter. Das Augenmerk wurde auf eine rein depressive Gruppe gelegt. Die Studie ist von der lokalen Ethikkommission (Ärztekammer Nordrhein) geprüft und akzeptiert worden und wurde unter der Nummer 2008331 registriert. Außerdem wurden in der Studie die Richtlinien der Deklaration von Helsinki [https://www. wma.net/policies-post/wma-declaration-of-helsinki-ethicalprinciples-for-medical-research-involving-human-subjects/] berücksichtigt.

\section{Design}

Ein Pragmatic Clinical Trial Design wurde angewendet [7]. Die klinische Intervention bestand aus 12 Wochen individualisierter Akupunkturbehandlung. Alle Patienten wurden über eine Akupunkturbehandlungsgruppe und eine Wartelistegruppe randomisiert. Für die Randomisierung wurde Excel benutzt (das Excel-Programm hat eine Funktion, die dazu erstellt wurde, dass sie Listen randomisiert, die Reihenfolge der Patienten kann demnach per Zufallsbefund eingeteilt werden; [8]). Bei allen Patienten wurde dreimal eine Stimmungsliste (BDI-II) erhoben, am Anfang der Studie, sofort nach der Akupunkturbehandlung/Wartelistekondition und nach drei Monaten. Darüber hinaus wurden alle Akupunkturpunkte, die in den Akupunkturbehandlungen benutzt wurden, analysiert. Es gab in dieser Studie klar abgegrenzte Funktionen pro Mitarbeiter. Die Testabnahmen wurden von Forschungsmitarbeitern (zwei Praktikanten) durchgeführt, die nicht über das Ziel der Studie informiert wurden. Ebenfalls wurde die Akupunktur "naiv“ durchgeführt und hatte lediglich das Ziel, die bestmöglichen Punkte pro Patient auszuwählen und eine Akupunkturbehandlung durchzuführen. Die Akupunkteurinnen hatten keine Information bezüglich der Phase der Studie und der Testverfahren. Die Statistik und die wissenschaftliche Arbeit wurden von anderen Forschern durchgeführt, die über das Ziel und die Hintergründe der Studie Bescheid wussten. 

Geschlechtsunterschiede bei der Akupunkturbehandlung von Patienten mit Depressionen

\section{Zusammenfassung}

Hintergrund Depression ist eine psychische Störung und zeichnet sich durch eine gedrückte, depressive Stimmung aus. Die Krankheit kommt häufiger bei Frauen als bei Männern vor. Meist werden Medikamente oder Psychotherapie als Behandlungsformen eingesetzt, aber heutzutage wird auch immer öfter Akupunktur als Zusatzbehandlung angewendet. Ziel der Arbeit Das Ziel dieser Studie ist zu untersuchen, ob es Geschlechtsunterschiede bei der Akupunkturbehandlung von Patienten mit Depressionen gibt.

Material und Methoden Es wurden 30 Patienten mit Depression, 20 Frauen und 10 Männer, an einer deutschen psychiatrischen Klinik untersucht. Ein Pragmatic Clinical Trial Design wurde benutzt, wo Patienten in eine Akupunkturbehandlungsgruppe und in eine Wartelistegruppe randomisiert zugeteilt wurden. Die klinische Intervention bestand aus insgesamt 12 Wochen individualisierter Akupunkturbehandlung. Alle Patienten wurden dreimal mithilfe einer Selbstratingskala (Beck-Depressions-
Inventar-II, BDI-II) getestet, und zwar am Anfang der Studie, sofort nach der Akupunkturbehandlung/Wartelistekondition und drei Monate danach. Auch wurden alle Akupunkturpunkte, die in den Behandlungen benutzt wurden, analysiert.

Ergebnisse Bei den Männern wurden mehr Nadeln gesetzt und mehr unterschiedliche Punkte verwendet. Bei der TCM-(Traditionelle-Chinesische-Medizin-)Diagnostik fiel auf, dass Frauen mehr Mangeldiagnosen zeigten und Männer mehr Fülle. Akupunktur verbesserte die Scores der Patienten (BDI-II) und diese Verbesserung blieb nach Beendigung der Behandlung stabil.

Schlussfolgerungen Die Resultate sollten in größeren Studien repliziert und weiter erforscht werden. Die Geschlechtsunterschiede sollten zukünftig besser im therapeutischen (Akupunktur-)Prozedere von Patienten mit Depressionen berücksichtigt werden.

Schlüsselwörter

Männer · Akupunkturtherapie · Depression · Frauen · Akupunkturpunkte

\section{Gender Differences in the Acupuncture Treatment of Patients with Depression}

\section{Abstract}

Background Depression is a mental disorder and is characterized by a low, depressive mood. The disorder is more frequent in women than in men. In most cases, medication or psychotherapy are used for treatment, but nowadays acupuncture is also increasingly used as an add-on treatment.

Objectives The aim of the present study was to investigate whether gender differences exist in the acupuncture treatment of patients with depression.

Materials and methods Thirty patients with depression, 20 females and 10 males, were recruited from a German psychiatric clinic. A pragmatic clinical trial design was used in which the patients were randomly divided into an acupuncture-treatment or a waiting-list group. The clinical intervention consisted of twelve weeks of individualized acupuncture treatment.
All patients were tested three times on a mood self-rating scale (Becks Depression Inventory-II, BDI-II) at the beginning of the study, after the acupuncture-treatment/waiting-list condition, and at follow-up. In addition, all acupuncture points that were used in the treatments were analyzed. Results Males received more needles and more points were used. The traditional Chinese medicine diagnostics showed that females have more emptiness and deficiency and males show more full patterns with heat. Acupuncture improved the score of the patients on the BDI-II and this improvement remained stable after finishing the treatment.

Conclusions In future acupuncture treatment of patients with depression, gender differences should be taken more into account. Moreover, the present results should be replicated.

Keywords

Male $\cdot$ Acupuncture therapy - Depression $\cdot$ Female $\cdot$ Acupuncture points

\section{Akupunkturintervention}

Die individuelle Akupunktur wurde nach individueller TCM(Traditionelle-Chinesische-Medizin-)Diagnose, inklusive Puls- und Zungendiagnostik [9], durch zwei lizenzierte TCMTherapeutinnen mit mehr als 5 Jahren klinischer Erfahrung angewendet und die STRICTA-Kriterien wurden angestrebt [10]. Welche Akupunkturpunkte bei welchen Mustern zur Anwendung kamen, wurde seitens der Akupunkteurinnen jeweils pro Behandlung festgelegt, es gab kein vorab feststehendes (semi)standardisiertes Protokoll. Die sterilen Einwegnadeln (AcuPro C, Wujiang City Cloud \& Dragon Medical Device Co., Ltd., China) für die Akupunkturbehandlung waren $0,25 \times 25 \mathrm{~mm}$ oder $0,20 \times 15 \mathrm{~mm}$ Edelstahlnadeln, abhängig von der Körperlokalisation. Sowohl bei der individuellen Akupunktur als auch bei der individuellen klinischen Diagnose wurde den Prinzipien der TCM gefolgt [11].

\section{Beck-Depressions-Inventar}

In der vorliegenden Studie wurde das Beck-Depressions-Inventar-II (BDI-II; [12]) als Fragebogen benutzt, um die Depressions- symptome der Patienten zu messen. Das BDI-II hat 21 Mehrfachauswahlfragen und die Versuchsperson muss aus vier Aussagen angeben, welche Antwort für ihn/sie in dieser Woche am zutreffendsten ist. Der totale Score liegt zwischen 0 und 63 Punkten, wobei 0-8 = keine Depression, 9-13 = minimale Depression, 14$19=$ leichte Depression, 20-28 = mittelschwere Depression und 29-63 = schwere Depression bezeichnen. Das BDI-II zeichnet sich durch eine exzellente Wiederholpräzision von 0,96 aus [13].

\section{Statistik}

Die SPSS-Version 22.0 [http://www-01.ibm.com/support/ docview.wss?uid=swg21646821] wurde für alle statistischen Analysen, wie $t$-Tests, Varianzanalysen usw., verwendet. Es fand für multiples Testen eine Korrektur mittels Bonferroni [14] statt. Das Signifikanzniveau von $p \leq 0,05$ wurde benutzt, um zu bestimmen, ob die Resultate signifikant sind. 
Tab. 1 Die weibliche Gruppe mit der westlichen Diagnose Depression

TCM-diagnostische Muster
Qi- \& Blut-Mangel
Nieren-Essenz-Mangel
Nieren-Yang-Mangel
Herz-Yin-Mangel
Blut-Mangel
Qi-Mangel
Herz-Feuer mit leerer Hitze
Nieren-Yin-Mangel
Leber- \& Nieren-Yin-Mangel mit Leber-Wind
Lungen- \& Milz-Qi-Mangel resultierend in Schleim, der den Kopf benebelt
Qi- \& Blut-Mangel resultierend in Schleim, der den Kopf benebelt

\begin{tabular}{|l|l|}
\hline Häufigkeit der Patientinnen (\%) & Absolute Zahl \\
\hline 35 & 7 \\
\hline 10 & 2 \\
\hline 10 & 2 \\
\hline 10 & 2 \\
\hline 5 & 1 \\
\hline 5 & 1 \\
\hline 5 & 1 \\
\hline 5 & 1 \\
\hline 5 & 1 \\
\hline 5 & 1 \\
\hline 5 & 1 \\
\hline
\end{tabular}

Tab. 2 Die männliche Gruppe mit der westlichen Diagnose Depression

TCM--diagnostische Muster
Schleim-Feuer
Herz-Blut-Hitze
Qi- \& Blut-Mangel
Nieren-Essenz-Mangel
Leber-Qi-Stase führend zu Leber-Blut-Stase
Leber-Feuer
Herz-Yin-Mangel

\begin{tabular}{|l|l|} 
Häufigkeit der Patienten (\%) & Absolute Zahl \\
\hline 30 & 3 \\
\hline 20 & 2 \\
\hline 10 & 1 \\
\hline 10 & 1 \\
\hline 10 & 1 \\
\hline 10 & 1 \\
\hline 10 & 1 \\
\hline
\end{tabular}

\section{Prozedur}

Die Patienten wurden in der Klinik in einem Zimmer individuell getestet. Zuerst bekamen sie von der Forschungsleiterin sowohl verbal als auch schriftlich generelle Instruktionen zur Studie und es wurden die Einwilligungsformulare unterschrieben. Außerdem wurden die Versuchspersonen eingehend darüber informiert, dass sie immer Fragen stellen können und dass sie jederzeit aus der Studie ohne Angabe von Gründen ausscheiden können. Danach bekamen sie die verbale und schriftliche Instruktion bezüglich des BDI-II. Die Versuchspersonen füllten den BDI-II-Fragebogen aus. Es gab 3 verschiedene Testzeitpunkte, eine Woche vor der Akupunkturbehandlung/Warteliste, eine Woche nach der Akupunkturbehandlung/Warteliste und 3 Monate nach Beendigung der Akupunkturbehandlung/Warteliste. Die Akupunkturbehandlung dauerte 12 Wochen und fand einmal für eine Dauer von 45 min wöchentlich statt. Am Ende der Studie wurden die Versuchspersonen nochmals über das Ziel der Studie informiert und es fand eine Nachbesprechung statt.

\section{Ergebnisse}

\section{Demografische Daten}

Die 30 Patienten litten im Durchschnitt 6 Jahre und 5 Monate unter chronischen Depressionen. Es gab keinen Unterschied in der durchschnittlichen Krankheitsdauer zwischen den 20 Frauen und 10 Männern $\left(F_{(1,29)}=0,648, p=0,427, \eta 2=0,023\right)$. Die Patienten waren im Mittel 47 Jahre und 6 Monate alt und es gab auch keinen Unterschied im Durchschnittsalter zwischen den 20 Frauen und 10 Männern $\left(F_{(1,29)}=1,437, p=0,241, \eta 2=0,049\right)$.

\section{TCM-Diagnostik}

Die Tab. 1 und 2 zeigen die diagnostischen TCM-Muster der Patienten mit Depressionen. Es gibt einige Geschlechtsunterschiede in Bezug auf die TCM-diagnostischen Muster. Die Frauen tendieren zu mehr Mangel als Männer und die Männer tendieren zu etwas mehr Fülle/Feuer als Frauen, außerdem fällt die große Heterogenität in beiden Gruppen auf.

\section{Wahl der Akupunkturpunkte}

In Abb. 1a ist zu sehen, dass bei den Patientinnen mit Depressionen am häufigsten die folgenden 8 Punkte genadelt wurden: EXHN-1 + Du Mai 20, Mi 6, Di 4, Mi 9, Ni 3, He 7, Ma 36 und Le 3. Aus der Abb. $1 \mathrm{~b}$ geht hervor, dass bei den männlichen Patienten mit Depressionen am häufigsten die folgenden 12 Punkte genadelt wurden: Di 4, EX-HN-1 + Du Mai 20, Ni 3, He 7, Di 11, Mi 9, Mi 6, Le 3, Ma 8, Ma 36, Mi 4 und KG 17. Obwohl die weiblichen und die männlichen Patienten mit Depressionen unter den häufigsten ( $>50 \%)$ Akupunkturpunkten 8 gleiche Punkte hatten, gab es auch deutliche Unterschiede, wie zum Beispiel die 4 zusätzlichen Punkte Di 11, Ma 8, Mi 4 und KG 17 bei den Männern. Es wurde generell bei den Behandlungen bei den weiblichen Patienten mit Depressionen festgestellt, dass etwas mehr unterschiedliche Akupunkturpunkte berücksichtigt wurden als bei den männlichen $\mathrm{Pa}$ tienten (54 versus 47). Bei den Patientinnen wurde pro Person 


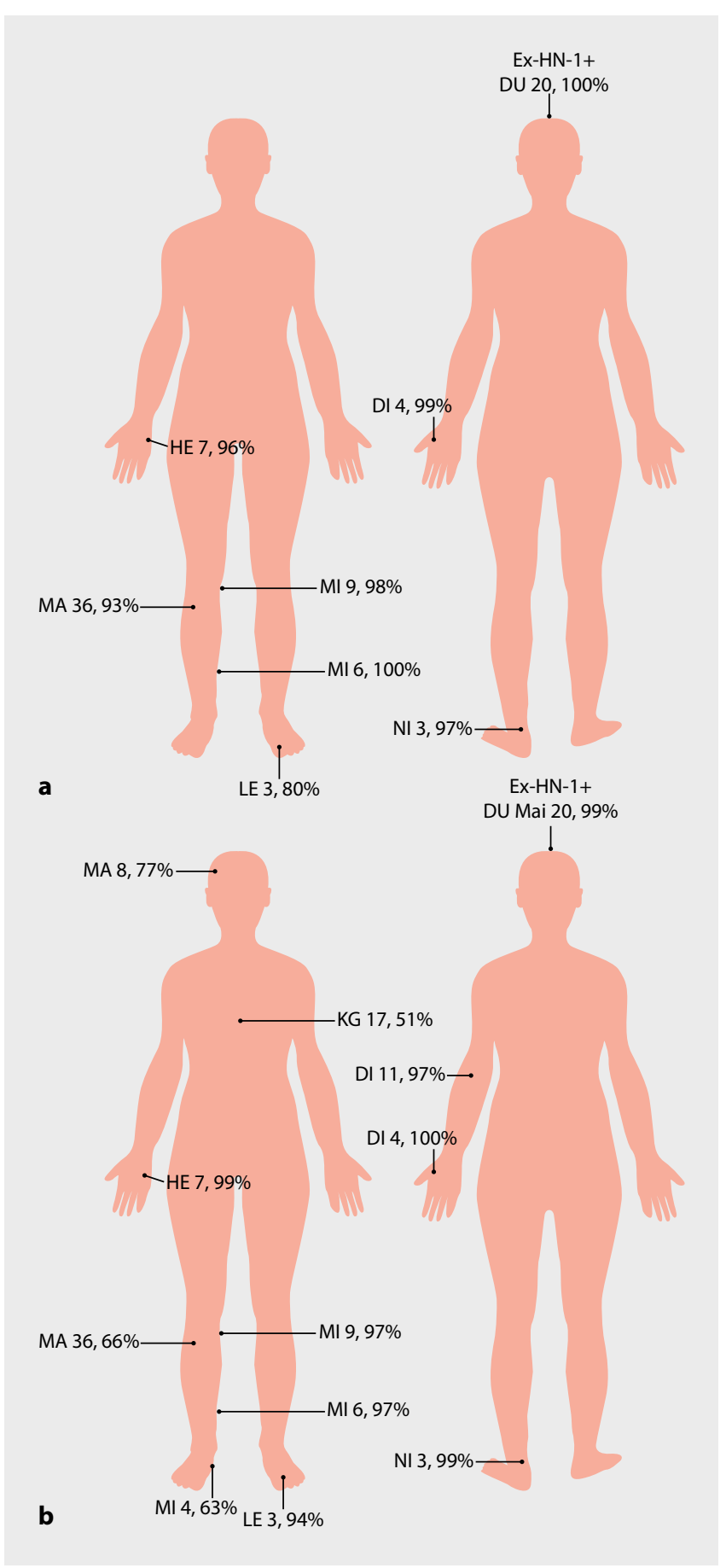

Abb. 1 a Die häufigsten ( $>50 \%$ ) Akupunkturpunkte, die bei der Behandlung der Frauen mit Depressionen benutzt wurden. b Die häufigsten (>50 \%) Akupunkturpunkte, die bei der Behandlung der Männer mit Depressionen benutzt wurden

etwa die gleiche Anzahl an unterschiedlichen Punkten genadelt wie bei den männlichen Patienten $(23,68$ Punkte $(S A=4,62)$ versus 24,00 Punkte $(S A=3,21))$. Beachtet man schließlich die Zahl der Nadeln, die pro Person pro Behandlung verwendet wurden, dann wurden bei den Patientinnen pro Person und pro Behandlung im Durchschnitt 28,35 $(S A=3,10)$ Nadeln verwendet und bei den männlichen Patienten 32,10 $(S A=4,00)$.

\section{BDI-II-Ergebnisse}

Die Resultate des BDI-II zeigen (Tab. 3 und 4), dass der Score nach der Akupunkturbehandlung niedriger ist als vor der Akupunkturbehandlung $\left(F_{(1,29)}=7,986, p=0,007, \eta^{2}=0,135\right)$ und dass es bei den Patienten in der Warteliste keine Veränderungen der Scores zwischen den zwei Messungen gegeben hat $\left(F_{(1,29)}=0,007\right.$, $\left.p=0,936, \eta^{2}=0,000\right)$. Drei Monate nach Beendigung der Akupunkturbehandlung sind die Werte stabil geblieben $\left(F_{(1,29)}=6,287\right.$, $\left.p=0,016, \eta^{2}=0,139\right)$. Die Resultate für das jeweilige Geschlecht (Tab. 3) zeigen getrennt betrachtet, dass die Akupunktureffekte bei den Frauen viel größer sind als bei den Männern. Bei den Frauen sieht man, dass die Scores nach der Akupunkturbehandlung signifikant niedriger sind als vor der Akupunkturbehandlung $\left(F_{(1,19)}=5,983, p=0,020, \eta^{2}=0,150\right)$, dass dies drei Monate nach Beendigung der Akupunkturbehandlung immer noch der Fall ist und dass die Werte sogar noch niedriger geworden sind $\left(F_{(1,13)}=10,749, p=0,003, \eta^{2}=0,301\right)$, während bei den Männern die Akupunktureffekte nicht signifikant sind. Wichtig ist hier allerdings zu erwähnen, dass es eine unterschiedliche Fallzahl gab (10 Männer versus 20 Frauen) und dass die nichtsignifikanten Resultate bei den Männern durch die viel kleinere Stichprobe erklärt werden können, denn die Abnahme war direkt nach der Akupunktur bei den Männern nominell größer als bei den Frauen.

\section{Diskussion}

Ziel dieser Studie war es zu untersuchen, ob es geschlechtsspezifische Unterschiede bei der Akupunkturbehandlung von Patienten mit Depressionen gibt. Die primäre Hypothese war, dass Geschlechtsunterschiede in den vorhandenen TCM-diagnostischen Mustern und in der Akupunkturbehandlung von weiblichen und männlichen Patienten mit Depressionen vorliegen. Die Analysen der TCM-diagnostischen Muster zeigen, dass in unseren Untersuchungsgruppen die weiblichen Patienten mit Depressionen viel mehr Mangelprobleme haben als Männer und die Männer mehr Fülle (Schleim und Feuer) zeigen als die Frauen, ansonsten gibt es kaum Geschlechtsunterschiede. Es fällt auf, dass eine große Heterogenität der Diagnosen bei beiden Gruppen vorhanden ist. Es ist wichtig darauf hinzuweisen, dass in der TCM individuelle Diagnostik benutzt wird und dass Geschlechtsunterschiede nicht speziell berücksichtigt werden. Es wäre interessant, dies in zukünftigen Studien gezielt zu untersuchen, da es auch praktische Implikationen haben könnte. Die Analyse der Punktauswahl zeigt, dass sowohl bei den weiblichen als auch bei den männlichen Patienten mit Depressionen am häufigsten die folgenden 8 Punkte genadelt werden: EX-HN-1 + Du Mai 20, Mi 6, Di 4, Mi 9, $\mathrm{Ni}$ 3, He 7, Ma 36 und Le 3. Eine Erklärung für die Verwendung von EX-HN-1 mit Du Mai 20 als auch He 7 und Le 3 sind deren bekannte starke Auswirkungen auf die Psyche, sie kalmieren das „Shen“ [15]. Diese Punkte wirken stark beruhigend und bei Patienten mit Depressionen, die regelmäßig über innere Unruhe, Grübeln und schlechtes Schlafen berichten, ist es logisch, dass diese Punkte Anwendung finden. Mi 6, Mi 9, Ma 36, Ni 3 und Di 4 sind wahrscheinlich die Punkte, die ohnehin am meisten benutzt werden, dies nicht zuletzt wegen ihrer bekannten Wirkung auf das Regulieren des Qis, die Regulierung des Wasserhaushalts und die Tonifizierung des Körpers [15]. Auch wenn man mit viel Mangelerscheinungen über den Nutzen von Le 3 diskutieren könnte, ist es so, dass dieser Punkt das Shen beruhigt und somit dem Geist Ruhe bereitet [15]. Darüber hinaus gab es einige Un- 
Tab. 3 Die Ergebnisse des BDI-II vor der Akupunkturbehandlung, unmittelbar nach der Akupunkturbehandlung und nach drei Monaten

\begin{tabular}{|c|c|c|c|}
\hline & BDI-II vor Akupunktur & BDI-II nach Akupunktur & BDI-II Follow-up \\
\hline Gesamte Gruppe & $27,73(9,61)^{\mathrm{a}}$ & $20,26(9,44)^{b}$ & $20,00(5,53)^{b}$ \\
\hline Frauen & $29,20(8,28)$ & $22,38(8,37)^{\mathrm{b}}$ & $18,29(4,75)^{b}$ \\
\hline Männer & $24,80(11,78)$ & $15,43(10,61)^{c}$ & $23,00(6,16)^{c}$ \\
\hline
\end{tabular}

Tab. 4 Die Ergebnisse des BDI-II der Warteliste bei der ersten Messung und bei der zweiten Messung

\begin{tabular}{l|l|l} 
& BDI-II erste Messung & BDI-II zweite Messung \\
\hline Gesamte Gruppe & $26,93(9,13)^{\mathrm{a}}$ & $26,67(12,55)^{\mathrm{b}}$ \\
\hline Frauen & $28,59(8,52)$ & $28,40(13,57)^{\mathrm{b}}$ \\
\hline Männer & $22,38(9,75)$ & $23,20(10,70)^{\mathrm{b}}$ \\
\hline aStandardabweichung, ${ }^{\text {b }}$ nichtsignifikant & &
\end{tabular}

terschiede zwischen den Frauen und den Männern, denn bei den Männern wurden die folgenden 4 Punkte Di 11, Ma 8, Mi 4 und KG 17 auch noch häufig genadelt. Die Anwendung dieser Punkte lässt sich aus der Häufigkeit der Hitzeerscheinungen bei den Männern erklären. Viele Männer hatten aufgrund der Hitzeprobleme Beschwerden mit Sodbrennen, wobei KG 17 häufig Anwendung fand [15]. Die Scores auf dem BDI-II, einer Stimmungsliste, die die Depressionssymptome der Patienten misst, sind nach der Akupunkturbehandlung für die totale Gruppe niedriger als vor der Akupunkturbehandlung und dies ist auch nach drei Monaten noch der Fall. Diese Ergebnisse stimmen mit den in früheren Studien gefundenen Resultaten überein $[16,17]$.

\section{》) Akupunktur scheint eine wirksame Form in der Behandlung von Depressio- nen zu sein}

Akupunktur scheint eine wirksame Behandlungsform in der Behandlung von Depressionen zu sein $[16,17]$. Die Resultate für jedes Geschlecht separat betrachtet zeigen, dass die Akupunktureffekte bei den Frauen viel größer sind als bei den Männern. Hier muss allerdings einschränkend angemerkt werden, dass die männliche Gruppe viel kleiner war (und eine hohe Standardabweichung hat) und sie deswegen auch viel weniger statistische Aussagekraft besitzt. Zweitens muss erwähnt werden, dass alle Patienten bereits unter Standardbehandlung standen und dass die Akupunkturbehandlung nur eine Zusatzbehandlung war. Vielleicht war die Standardbehandlung bei den Männern schon etwas erfolgreicher, da sie bereits zu Beginn der Studie ein niedrigeres Ausgangsniveau im BDI-II-Score aufwies.

In der Literatur gibt es bis jetzt lediglich einige Akupunkturstudien, die die geschlechtsspezifischen Unterschiede näher untersucht haben $[18,19]$. Es wurde gefunden, dass es tatsächlich Unterschiede zwischen Männern und Frauen, beispielsweise in der Reaktion auf (Laser-)Akupunktur, gibt [18]. Auch hinsichtlich der Hirnaktivität in Reaktion auf Akupunktur gibt es solche Nachweise [19] und diese Studien beschreiben beide stärkere Effekte bei Frau- en $[18,19]$. Darüber hinaus ist es hier wichtig zu erwähnen, dass es aus der Literatur bekannt ist, dass die Symptomatik depressiver Störungen bei Frauen und Männern unterschiedlich ausgeprägt ist [3].

Die vorliegende Studie hat einige Limitierungen, wie zum Beispiel die kleine Stichprobe [11], besonders die geringe Zahl an Männern (es ist üblich, dass mehr Frauen als Männer an Depression leiden [2]) und die Tatsache, dass die Patienten aus ethischen Gründen während der Studie alle weiterhin unter medikamentöser Behandlung standen [11]. Eine dritte Limitierung ist, dass nur ein Selbstratingfragebogen (BDI-II [12]) angewandt wurde und keine Fremdratings, wie zum Beispiel die Hamilton Skala [20], bei der von einem Untersucher beurteilt wird, wie schwer ein bestimmtes Symptom ausgeprägt ist. Auch gab es keine Verblindung [21] in dieser Studie, deshalb müssen die Ergebnisse mit Vorsicht betrachtet werden [22]. Eine fünfte Limitierung ist das Faktum, dass die Patientengruppe mit Depressionen, obwohl strikten klinischen Kriterien [1] gefolgt wurde, heterogen war. Dies ist für die westliche medizinische Diagnostik ein Problem, aber besonders auch für die östliche [23]. Dieses methodologische Problem ist in der Akupunkturforschungsliteratur bekannt als das „Obstkorbproblem“ [24]. Es beschreibt die Problematik, dass obwohl die Patienten nach westlicher Medizin alle mit „Depression“ diagnostiziert werden, sie nach östlicher Medizin ganz verschiedene diagnostische Muster zeigen [24]. Deswegen ist es auch keine große Überraschung, dass die Akupunkturergebnisse in einer Patientengruppe mit Depressionen so unterschiedlich sind. Wegen dieser methodologischen Limitierungen müssen die Ergebnisse und Schlussfolgerungen dieser Arbeit mit Vorsicht betrachtet werden, da sie in zukünftigen Studien noch mit größeren Stichproben und besseren methodologischen Verfahren repliziert werden müssen. Außerdem wäre es empfehlenswert, die verschiedenen östlichen diagnostischen Muster, neben der westlichen Diagnose, zu beschreiben [25]. In einer sogenannten Big-Data-Studie könnte man die Daten darüber hinaus auch nach verschiedenen östlichen medizinischen diagnostischen Mustern analysieren [25].

\section{Schlussfolgerung}

Die Studie zeigt einen Trend dahin gehend, dass bei geschlechtsspezifischer Betrachtung unterschiedliche TCM-Diagnosen erstellt werden und daher andere Behandlungen notwendig sind. 
Möglicherweise ist die Reaktion auf Akupunktur auch eine unterschiedliche. Diese vorläufigen Ergebnisse sollten in zukünftigen Big-Data-Studien untersucht werden.

\section{Fazit für die Praxis}

Patienten mit Depressionen werden in der Praxis bei Akupunkturbehandlungen nach östlichen medizinischen Mustern behandelt. Es handelt sich dann um eine individuelle klinische Diagnostik. Wegen dieses individuellen Ansatzes werden die Geschlechtsunterschiede nicht speziell berücksichtigt. Die aktuellen Akupunkturstudien zeigen allerdings, dass es geschlechtsspezifische Unterschiede in der Reaktion auf Akupunktur gibt, die auch in der Praxis bei der Punkteauswahl bei der Behandlung von Patienten mit Depressionen besser berücksichtigt werden müssen.

Literatur

1. World Health Organization (1992) The ICD-10 classification of mental and behavioural disorders: Clinical descriptions and diagnostic guidelines. WHO, Genf

2. Albert PR (2015) Why is depression more prevalent in women? J Psychiatry Neurosci 40(4):219-221. https://doi.org/10.1503/jpn.150205

3. Möller-Leimkühler AM (2009) Männer, Depression und „männliche Depression”. Fortschr Neurol Psychiatr 77(7):412-419. https://doi.org/10.1055/s-2008-1038257 (quiz 420)

4. Kupfer DJ (2005) The pharmacological management of depression. Dialogues Clin Neurosci 7(3):191-205

5. Health Quality Ontario (2017) Psychotherapy for major depressive disorder and generalized anxiety disorder: A health technology assessment. Ont Health Technol Assess Ser 17(15):1-167

6. Bosch P, van den Noort M, Staudte H, Lim S (2015) Schizophrenia and depression: A systematic review of the effectiveness and the working mechanisms behind acupuncture. Explore (NY) 11(4):281-291. https://doi.org/10.1016/j. explore.2015.04.004

7. MacPherson H (2004) Pragmatic clinical trials. Complement Ther Med 12(2/3):136140. https://doi.org/10.1016/j.ctim.2004.07.043

8. Microsoft (2015) Microsoft Excel 2016. Step by Step

9. Bosch P, Staudte H, Yeo S, Lee S-H, Lim S, van den Noort M (2017) Acupuncture treatment of a male patient suffering from long-term schizophrenia and sleep disorders. J Tradit Chin Med 37(6):862-867. https://doi.org/10.1016/502546272(18)30052-9

10. MacPherson H, Altman DG, Hammerschlag R, Youping L, Taixiang W, White A, Moher D, STRICTA Revision Group (2010) Revised standards for reporting interventions in controlled trials of acupuncture: Extending the CONSORT statement. Plos Med 7:e1000261. https://doi.org/10.1371/journal.pmed.1000261

11. Bosch P, van Luijtelaar G, van den Noort M, Lim S, Egger J, Coenen A (2013) Sleep ameliorating effects of acupuncture in a psychiatric population. Evid Based Complement Alternat Med. https://doi.org/10.1155/2013/969032

12. Beck AT, Steer RA, Brown GK (1996) Manual for the Beck depression inventory-II. Psychological Corporation, San Antonio

13. Sprinkle SD, Lurie D, Insko SL, Atkinson G, Jones GL, Logan AR, Bissada NN (2002) Criterion validity, severity cut scores, and test-retest reliability of the Beck depression inventory-ll in a university counseling center sample. J Couns Psychol 49(3):381-385. https://doi.org/10.1037/0022-0167.49.3.381

14. Huberty CJ, Morris JD (1989) Multivariate analysis versus multiple univariate analyses. Psychol Bull 105(2):302-308. https://doi.org/10.1037/0033-2909.105.2.302

15. Cheng X (1987) Chinese acupuncture and moxibustion. Foreign Languages Press, Beijing

16. Kramer S (2018) Studienlage zu Akupunktur bei Depressionen. Dtsch Z Akupunktur 61(1):9-11. https://doi.org/10.1007/s42212-018-0023-1
17. MacPherson H, Richmond S, Bland M, Brealey S, Gabe R, Hopton A, Keding A, Lansdown H, Perren S, Sculpher M, Spackman E, Torgerson D, Watt I (2013) Acupuncture and counselling for depression in primary care: A randomised controlled trial. Plos Med 10(9):e1001518. https://doi.org/10.1371/journal.pmed.1001518

18. Litscher D, Wang J, Litscher G, Li G, Bosch P, van den Noort M, Wang L (2018) Gender differences in laser-acupuncture - Results of a crossover study with green and yellow laser at the ear point Shenmen. Medicines (Basel) 5(1):E24. https://doi.org/10.3390/ medicines 5010024

19. Yeo S, Rosen B, Bosch P, Noort MV, Lim S (2016) Gender differences in the neural response to acupuncture: Clinical implications. Acupunct Med 34(5):364-372. https:// doi.org/10.1136/acupmed-2015-011025

20. Hamilton M (1960) A rating scale for depression. J Neurol Neurosurg Psychiatry 23:56-62. https://doi.org/10.1136/jnnp.23.1.56

21. Allen JJ, Schnyer RN, Chambers AS, Hitt SK, Moreno FA, Manber R (2006) Acupuncture for depression: A randomized controlled trial. J Clin Psychiatry 67(11):1665-1673

22. Wu J, Yeung AS, Schnyer R, Wang Y, Mischoulon D (2012) Acupuncture for depression: A review of clinical applications. Can J Psychiatry 57(7):397-405. https://doi. org/10.1177/070674371205700702

23. Bosch P, de Rover P, Staudte H, Lim S, van den Noort M (2015) Schizophrenia, depression, and sleep disorders: Their traditional Oriental medicine equivalents. J Acupunct Meridian Stud 8(1):17-22. https://doi.org/10.1016/j.jams.2014.06.001

24. Bosch P, de Rover P, Yeo S, Lee SH, Lim S, van den Noort M (2016) Traditional Chinese medicine in psychiatry: The fruit-basket-problem. J Integr Med 14(4):239-240. https://doi.org/10.1016/S2095-4964(16)60258-3

25. van den Noort M, Yeo S, Lim S, Lee SH, Staudte H, Bosch P (2018) Acupuncture as add-on treatment of the positive, negative, and cognitive symptoms of patients with schizophrenia: A systematic review. Medicines (Basel) 5(2):E29. https://doi. org/10.3390/medicines5020029

\section{Korrespondenzadresse}

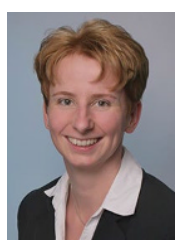

Dr. Peggy Bosch

Psychiatrische Forschungsgruppe, LVR-Klinik Bedburg-Hau

Bahnstraße 6, 47551 Bedburg-Hau, Deutschland Margaretha.Bosch@lvr.de

Danksagung. In Österreich wurden die wissenschaftlichen Untersuchungen vom Bundesministerium für Bildung, Wissenschaft und Forschung (,,SinoAustrian TCM Research on Lifestyle-related Diseases: Innovative Acupuncture Research" (2016-2019); Projektleiter Gerhard Litscher) unterstützt.

\section{Einhaltung ethischer Richtlinien}

Interessenkonflikt. P. Bosch, S. Lim, H. Staudte, S.-H. Lee, S. Yeo, D. Litscher, G. Litscher, L. Wang und M. van den Noort geben an, dass kein Interessenkonflikt besteht.

Alle beschriebenen Untersuchungen am Menschen oder an menschlichem Gewebe wurden mit Zustimmung der zuständigen Ethikkommission, im Einklang mit nationalem Recht sowie gemäß der Deklaration von Helsinki von 1975 (in der aktuellen, überarbeiteten Fassung) durchgeführt. Von allen beteiligten Patienten liegt eine Einverständniserklärung vor.

Open Access. Dieser Artikel wird unter der Creative Commons Namensnennung 4.0 International Lizenz (http://creativecommons. org/licenses/by/4.0/deed.de) veröffentlicht, welche die Nutzung, Vervielfältigung, Bearbeitung, Verbreitung und Wiedergabe in jeglichem Medium und Format erlaubt, sofern Sie den/die ursprünglichen Autor(en) und die Quelle ordnungsgemäß nennen, einen Link zur Creative Commons Lizenz beifügen und angeben, ob Änderungen vorgenommen wurden. 